\title{
Cellular automaton model for the simulation of laser dynamics
}

\author{
J. L. Guisado, ${ }^{1, *}$ F. Jiménez-Morales, ${ }^{1, \dagger}$ and J. M. Guerra ${ }^{2,}$ \\ ${ }^{1}$ Departamento de Física de la Materia Condensada, Universidad de Sevilla, P.O. Box 1065, 41080 Sevilla, Spain \\ ${ }^{2}$ Departamento de Optica, Facultad de CC. Físicas, Universidad Complutense de Madrid, 28040 Madrid, Spain
}

(Received 28 November 2002; published 20 June 2003)

\begin{abstract}
The classical modeling approach for laser study relies on the differential equations. In this paper, a cellular automaton model is proposed as an alternative for the simulation of population dynamics. Even though the model is simplified it captures the essence of laser phenomenology: (i) there is a threshold pumping rate that depends inversely on the decaying lifetime of the atoms and the photons; and (ii) depending on these lifetimes and on the pumping rate, a constant or an oscillatory behavior can be observed. More complex behaviors such as spiking and pattern formation can also be studied with the cellular automaton model.
\end{abstract}

DOI: 10.1103/PhysRevE.67.066708

PACS number(s): 07.05.Tp, 42.55.Ah, 42.60.Jf, 42.65.Sf

\section{INTRODUCTION}

In a laser system, the interactions among simple atoms and the radiation they produce can give rise to cooperative phenomena [1]. However, the usual approach for its study is based on very detailed microscopical equations, which somehow mask the action of such cooperative properties. In this study, a simple cellular automaton model is presented, which reproduces much of the laser phenomenology, special attention being focused on these self-organizing cooperative effects. Our model is interesting in that it illustrates the emergence of laser properties as cooperative phenomena based on simple underlying rules. It can also be useful in calculating laser output in situations which are difficult to treat with the traditional approach based on the resolution of detailed differential equations [2,3]. One example is when dealing with complex boundary conditions or numerical difficulties in the integration of the equations.

Cellular automata $[4,5]$ are a class of spatially and temporally discrete mathematical systems, characterized by local interaction and synchronous dynamical evolution. They have the ability to generate a very complex behavior from sets of components that interact locally with relatively simple rules. They provide good models for a wide variety of physical systems $[6,7]$ exhibiting cooperative phenomena, such as magnetization in solids [8], reaction-diffusion processes [9], fluid dynamics for complex situations [10], growth phenomena [11], etc. As an example of an application particularly close to laser dynamics, a cellular automaton model has been recently applied to successfully study the excited-state dynamics of atomic oxygen, which play a prominent role in creating the aurora borealis [12].

This paper is organized as follows. Section II presents a brief review of laser dynamics including the laser phenomenology that our model attempts to reproduce. Section III describes the cellular automaton model. The simulations are

\footnotetext{
* Present address: Centro Universitario de Mérida, Universidad de Extremadura, 06800 Mérida (Badajoz), Spain. Electronic address: jlguisado@unex.es

${ }^{\dagger}$ Electronic address: jimenez@us.es

†Electronic address: jmguerra@ fis.ucm.es
}

carried out and their results are presented in Sec. IV. Finally, the conclusions of this study are explained in Sec. V.

\section{LASER DYNAMICS}

A laser is a device that generates or amplifies electromagnetic radiation based on the stimulated emission phenomenon. The basic components of a laser system are (1) a laser medium - an appropriate collection of atoms, molecules, ions or a semiconductor crystal (these elements will generally be referred to as "atoms"); (2) a pumping process that excites electrons from these atoms to upper energy levels, due to some external electrical, optical, or chemical energy source; (3) optical feedback elements that reflect repeatedly the radiation beam into the laser medium (in a laser oscillator), or allow it to pass only once through it (in a laser amplifier).

The working principle of laser is stimulated emission, i.e., an excited atom can decay to a lower state stimulated by the presence of a photon with energy equal to the difference between the two energy levels, emitting a second photon with the same frequency and propagating in the same direction. The process of absorption has the same probability, so stimulated emission dominates only when a population inversion is induced in the material by some pumping mechanism.

A simplified but yet realistic model of many real lasers is the four-level laser system shown in Fig. 1. The population dynamics of a laser (the variation with time in the number of laser photons and in the population inversion, or number of electrons in the upper laser level minus the number of electrons in the lower laser level) is usually described as a system of coupled differential equations called laser rate equations.

The rate equations can be put into their simplest forms when the lifetimes of $E_{1}$ and $E_{3}$ level electrons are negligible as compared to the lifetime of $E_{2}$ level electrons. Therefore the $E_{1}$ level electron population is $N_{1} \simeq 0$, and thus the population inversion is approximately equal to the upper laser level population $N(t)=N_{2}(t)-N_{1}(t) \simeq N_{2}(t)$; and the absorption of laser photons by electrons at level $E_{1}$ is negligible. Additionally, the pumping into level $E_{2}$ can be described by a simple pumping rate $R$.

Under these assumptions, which are quite realistic, only 


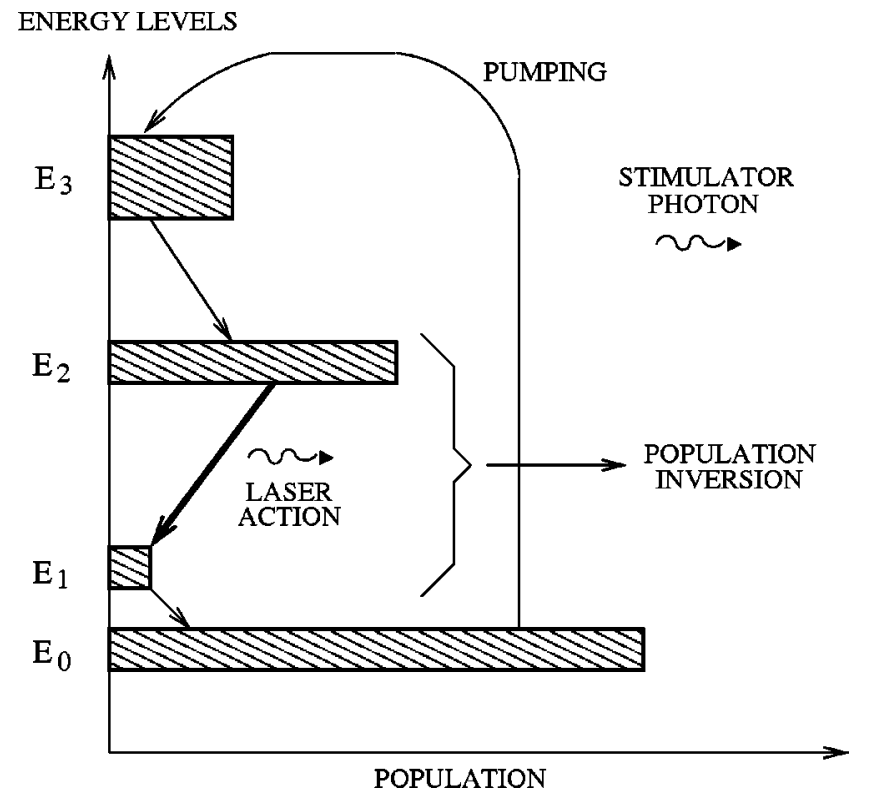

FIG. 1. Schematic view of a four-level laser system.

levels $E_{0}$ and $E_{2}$ can be considered, and the rate equations are a system of two coupled differential equations $[2,3]$ :

$$
\begin{gathered}
\frac{d n(t)}{d t}=K N(t) n(t)-\frac{n(t)}{\tau_{c}}, \\
\frac{d N(t)}{d t}=R-\frac{N(t)}{\tau_{a}}-K N(t) n(t) .
\end{gathered}
$$

Here $R$ is the pumping rate, $\tau_{a}$ is the decay time of the upper laser level $\left(E_{2}\right), \tau_{c}$ is the decay time of the laser photons in the cavity, and $K$ is a constant called "coupling constant." The first equation reflects the variation with time of the number of laser photons, $n(t)$, which is related to the laser beam intensity. The second equation represents the temporal variation of the population inversion $N(t)$. This is a set of two coupled nonlinear equations due to the presence of the product term $K N(t) n(t)$.

Laser operation does not generally produce a smooth and continuous response, but exhibits different kinds of characteristic transient or modulation behaviors such as spiking, relaxation oscillation, gain switching. Laser spiking, in particular, refers to the pulses that typically occur during the initial turn-on phase of many lasers. In these cases, the laser signal presents a sequence of sharp, large-amplitude narrow pulses or "spikes." Each spike is typically a fraction of a microsecond wide, and they are separated by a few microseconds. In some lasers, the spiking behavior jumps in an erratic way. In other cases, or under more stable conditions, it is possible to obtain a more regular spiking behavior, in which the amplitude of the spikes gradually damps out with time into a relaxation process. These are sometimes called relaxation oscillations, and include lasers with largeamplitude oscillations when enough time has elapsed for the amplitude to relax. This kind of behavior varies for the different types of lasers. The spiking oscillatory response is characteristic of most solid-state and semiconductor lasers, which have a substantially longer decay time of the upper laser level than the decay time of the photons in the laser cavity: $\tau_{a} \gg \tau_{c}$. Laser spiking and relaxation oscillations are not observed, on the other hand, in most gas lasers, which usually have decay times of the same order of magnitude, $\tau_{a} \approx \tau_{c}$.

The steady-state solution to the rate equations above the threshold (i.e., with laser emission) is

$$
\begin{gathered}
N_{s}=\frac{1}{K \tau_{c}}, \\
n_{s}=\tau_{c}\left(R-\frac{1}{K \tau_{a} \tau_{c}}\right) .
\end{gathered}
$$

In order for this solution to have a physical meaning, the number of photons, $n$, has to be greater than zero. The minimum value of $R$ producing a laser emission in the cavity, known as threshold pumping rate $R_{t}$ is found as

$$
R_{t}=\frac{1}{K \tau_{a} \tau_{c}} .
$$

For the case when the spiking behavior in the laser has relaxed to small-amplitude fluctuations, a linearized smallsignal analysis of the rate equations about the steady-state solution can be carried out. Following two different situations arise.

(a) Nonspiking lasers: For lasers in which $\tau_{a} \approx \tau_{c}$, the solutions for $n(t)$ and $N(t)$ are real exponentials. The system is overdamped and the response of the laser to any perturbation dies out exponentially towards the steady state. The solution is a stable node.

(b) Relaxation oscillations: When $\tau_{a} \gg \tau_{c}$ the solutions have an exponentially damped sinusoidal form towards the steady-state values. The system responds to any perturbation exhibiting relaxation oscillations. The solution is a stable focus. The relaxation-oscillation frequency, often referred to as spiking frequency, is found as

$$
\omega_{s p}=\sqrt{\left(\frac{1}{2 \tau_{a}} \frac{R}{R_{t}}\right)^{2}-\frac{1}{\tau_{a} \tau_{c}}\left(\frac{R}{R_{t}}-1\right)} .
$$

The necessary condition for the appearance of the relaxation oscillations is that the quantity inside the square root be positive, yielding

$$
\frac{\tau_{a}}{\tau_{c}}>\frac{\left(\frac{R}{R_{t}}\right)^{2}}{4\left(\frac{R}{R_{t}}-1\right)} .
$$

\section{CELLULAR AUTOMATA MODEL}

Cellular automata (CA) are fully discrete dynamical systems, where the states are chosen in a finite set and distributed on a discrete regular lattice, and the time evolution is run synchronously in all the sites of this lattice and each site 
changes its state according to a local rule that only depends upon its neighboring values. Despite the simplicity of their construction, CA are found to be capable of a diverse and complex behavior and are often used as a prototype for the analysis of the spontaneous emergence of ordered behavior in spatially extended systems that are locally coupled.

\section{A. States of the cells}

We consider a system composed of a square lattice of $N_{c}=L \times L$ cells with periodic boundary conditions. Two variables $a_{i}(t)$ and $c_{i}(t)$ are associated to each node of this lattice. The first one, $a_{i}(t)$, represents the state of the electron in node $i$ at a given time $t$. An electron in the laser ground state takes the value $a_{i}(t)=0$, while an electron in the upper laser state takes the value $a_{i}(t)=1$. A temporal variable $\tilde{a}_{i}(t) \in\left\{0,1,2, \ldots, \tau_{a}\right\}$ is also introduced in order to take into account the finite time $\tau_{a}$, for which an electron can remain in the upper state. If the electron is in the base state, then $\tilde{a}_{i}(t)=0$, otherwise $\tilde{a}_{i}(t+1)=\tilde{a}_{i}(t)+1$ until the maximum value $\tau_{a}$ is reached and then $\tilde{a}_{i}(t+1)=0$.

The second variable $c_{i}(t) \in\{0,1,2, \ldots, M\}$ represents the number of photons in node $i$ at time $t$. A large enough upper value of $M$ is taken to avoid saturation of the system. There is also another temporal variable $\tilde{c}_{i}^{j}(t) \in\left\{0,1,2, \ldots, \tau_{c}\right\}$, which measures the amount of time since a photon $j$ $\in\{1,2, \ldots, M\}$ was created at node $i . \tau_{c}$ is the lifetime of each photon. For a given photon $j, \tilde{c}_{i}^{j}(t+1)=\tilde{c}_{i}^{j}(t)+1$ until the lifetime $\tau_{c}$ is reached and then $\tilde{c}_{i}^{j}(t+1)=0$.

\section{B. Neighborhood}

The neighborhood considered is the Moore neighborhood, each cell having nine neighbors: the cell itself, its four nearest neighbors (situated in the positions north, south, east, and west), and the four next neighbors (in the positions northeast, southeast, northwest, and southwest).

\section{Transition rules}

The time evolution of the CA is given by a set of rules which determines the state of any particular cell of the system at time $t+1$, depending on the state of the cells included in its neighborhood at time $t$. The evolution of the temporal variables $\tilde{a}_{i}(t)$ and $\tilde{c}_{i}^{j}(t)$ was described beforehand. Here we describe only the evolution of $a_{i}(t)$ and $c_{i}(t)$.

(i) If $a_{i}(t)=0$, then

$$
a_{i}(t+1)=1 \quad \text { with probability } \lambda .
$$

(ii) If $a_{i}(t)=1$ and $\Gamma_{i}(t)=\Sigma_{\text {neighbors }} c_{i}(t)>\delta$, then

$$
\begin{gathered}
c_{i}(t+1)=c_{i}(t)+1, \\
a_{i}(t+1)=0,
\end{gathered}
$$

where $\Gamma_{i}$ is the number of photons included in the neighborhood of the cell $i$ and $\delta$ is a threshold value, which has been taken to be 1 in our simulations. (iii) If $c_{i}(t)>0$ and there is one photon $j$ for which $\tilde{c}_{i}^{j}(t)=\tau_{c}$, then

$$
c_{i}(t+1)=c_{i}(t)-1 .
$$

(iv) If $a_{i}(t)=1$ and $\tilde{a}_{i}(t)=\tau_{a}$, then

$$
a_{i}(t+1)=0 .
$$

These transition rules represent the different physical processes that function at the microscopical level in a laser system. Rule (i) represents the pumping process. Rule (ii) models the stimulated emission-if the electronic state of a cell has a value of $a_{i}(t)=1$ and the number of laser photons in the neighborhood is greater than a certain threshold, then at the time $t+1$ a new photon will be created in that cell and the electron will decay to the ground level. Rule (iii) represents the photon decay. Rule (iv) represents the electron decay in a way similar to rule (iii) —after time $\tau_{a}$ of the excited electrons, those electrons will decay to the ground level. To simplify the model as much as possible, we consider this decay to be entirely nonradiative, i.e., spontaneous emission is not taken into account. Also, as in an ideal four-level laser the population of level $E_{1}$ is negligible, stimulated absorption has not been considered.

Additionally, in order to represent the noise level observed in practice (typically of the order of one noise photon per cavity mode), we introduce a small continuous noise level of random photons in the laser mode at every time step. This is done by setting $c_{i}(t+1)=c_{i}(t)+1$ for a number of cells smaller than $0.01 \%$ of the total number of system cells, whose positions are randomly chosen. This noise (along with the population inversion induced by pumping) is responsible for the initial start up of the laser process, and can prevent the eventual extinction of this process, in case the number of laser photons drops down to negligible values.

\section{SIMULATIONS}

The simulations were carried out using lattices of 200 $\times 200$ and $300 \times 300$ cells. Three parameters determine the response of the system: the pumping probability $\lambda$, the lifetime of photons $\left(\tau_{c}\right)$, and the lifetime of excited electrons $\left(\tau_{a}\right)$. The pumping probability $\lambda$ introduced in the CA model is linearly related to the constant pumping rate $R$ included in the rate equations, as explained in the Appendix. Thus the laser rate equations' prediction [Eq. (5)] for the dependence of threshold pumping rate $R_{t}$ with the lifetimes $\tau_{a}$ and $\tau_{c}$ can be expressed for the threshold pumping probability $\lambda_{t}$ as

$$
\lambda_{t}=\frac{1}{K^{\prime} \tau_{a} \tau_{c}}
$$

where $K^{\prime}$ is a constant.

Figure 2 shows the threshold pumping probability $\lambda_{t}$ versus the lifetime of the upper laser level $\left(\tau_{a}\right)$ and the cavity lifetime $\tau_{c}$. Each point has been obtained in the following manner. After a transient time, the system evolves for 200 time steps and the average number of laser photons $(\bar{n})$ is 
(a)

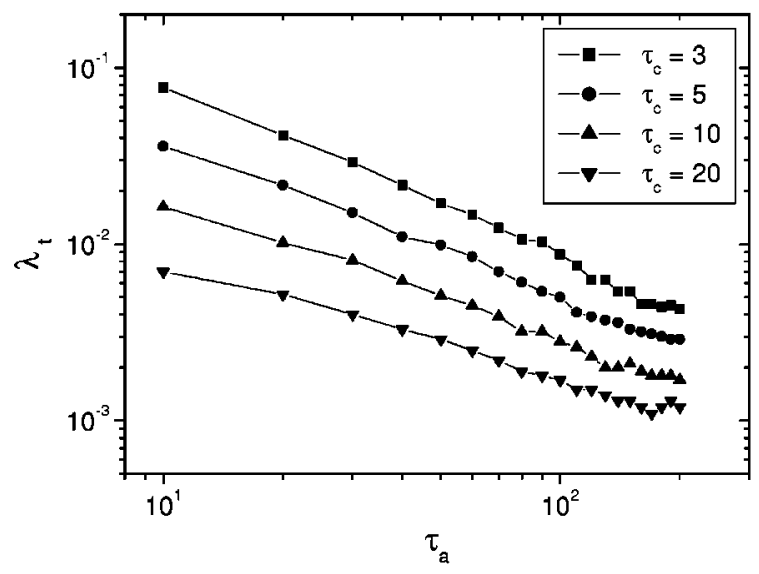

(b)

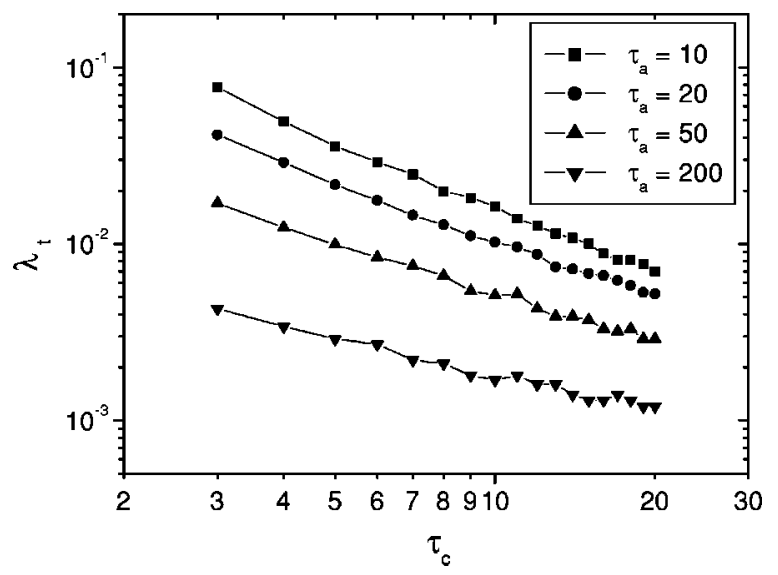

FIG. 2. Threshold pumping probability $\lambda_{t}$ from the CA laser model: (a) $\lambda_{t}$ dependence on the upper laser level lifetime $\tau_{a}$ for different values of the cavity lifetime $\tau_{c}$; (b) $\lambda_{t}$ dependence on $\tau_{c}$ for different values of $\tau_{a}$. Both figures are plotted on a logarithmic scale. $\tau_{a}$ and $\tau_{c}$ are measured in time steps.

recorded. For each pair of values $\tau_{c}$ and $\tau_{a}$, the procedure is repeated for values of the pumping probabilities $\lambda$ ranging from 0.0001 to 0.1 . If the laser action does not initiate in the system, $\bar{n}$ is approximately equal to the number of noise photons $\left(n_{n p}\right)$ introduced. When the laser action starts, photons are produced by stimulated emission [via rule (ii)], and the average number of photons is greater than $n_{n p}$. Thus, $\lambda_{t}$ can be computed as the minimum value of the pumping probability for which the average number of photons is higher than a given reference value $n_{\min }$. In our simulations, we have taken $n_{\min }=1.25 n_{n p}$. The number of noise photons $\left(n_{n p}\right)$ is the number of noise photons introduced in each time step multiplied by the lifetime of each laser photon.

Figure 3 shows the threshold pumping probability $\lambda_{t}$ versus $\tau_{a} \tau_{c}$ on a logarithmic scale. It can be seen that all the different curves of Fig. 2 collapse in a unique straight line, having a slope close to -1 , which is in agreement with the behavior predicted by the laser rate equations, i.e., Eq. (8).

To analyze the different dynamic behaviors of the system, we allow it to evolve for 500 time steps. Initially all the electrons are in the ground level $\left[a_{i}(0)=0\right]$ and there are no

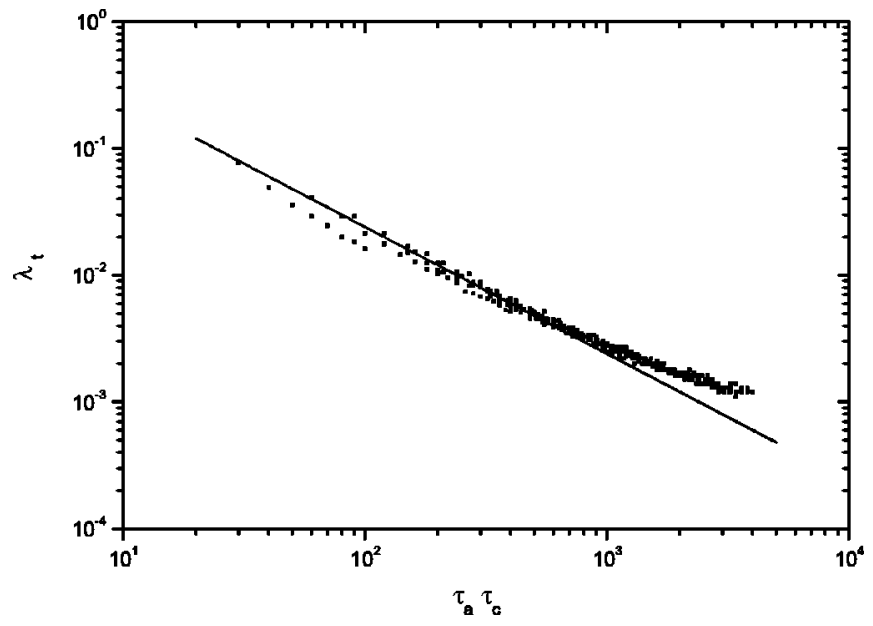

FIG. 3. Dependence of the threshold pumping probability $\lambda_{t}$ from the CA laser model versus $\tau_{a} \tau_{c}$, plotted on a logarithmic scale. The dots correspond to the results of the simulations and the continuous line to the theoretical prediction of the laser rate equations, i.e., Eq. (8). $\tau_{a}$ and $\tau_{c}$ are measured in time steps.

laser photons present $\left[c_{i}(0)=0\right]$, except a small fraction $(0.01 \%)$ of noise photons. In each experiment, the total number of laser photons, $n(t)=\sum_{i=1}^{N_{c}} c_{i}(t)$, and the total number of electrons in the upper laser state (population inversion), $N(t)=\sum_{i=1}^{N_{c}} a_{i}(t)$ are measured. In order to classify the range of values of the parameters for which oscillations or a constant regime in $n(t)$, and $N(t)$ appear, the Shannon entropy $S$ of the distribution of values taken by $n(t)$ and $N(t)$ is calculated. This is carried out by dividing the range of values taken in $10^{3}$ intervals, and computing the frequency $f_{i}$ at which the magnitude value lies inside every particular nonvoid interval $i$. The Shannon entropy is expressed as

$$
S\left(\lambda, \tau_{c}, \tau_{a}\right)=-\sum_{i=1}^{m} f_{i} \log _{2} f_{i},
$$

where $m$ is the number of nonvoid intervals. Figure 4 shows the contour plot of the Shannon entropy of the values distribution of the number of laser photons, $S_{c}$, for a fixed value of $\tau_{c}=10$. Similar plots are obtained for other values of $\tau_{c}$. The black line is the theoretical stability curve:

$$
\frac{\tau_{a}}{\tau_{c}}=\frac{\left(\frac{R}{R_{t}}\right)^{2}}{4\left(\frac{R}{R_{t}}-1\right)},
$$

which follows from Eq. (7). Here $R / R_{t}$ is used instead of $\lambda / \lambda_{t}$, because Eq. (A5) from the Appendix, where $\left(\lambda / \lambda_{t}\right)$ $=\left(R / R_{t}\right)$, was taken into account. The Shannon entropy measures the dispersion in the distribution of the number of laser photons (or in the population inversion). If this number is approximately constant, $S$ will tend toward zero; if oscillations appear, $S$ takes higher values. The maximum value would result from an equiprobable distribution. $S$ is thus a 


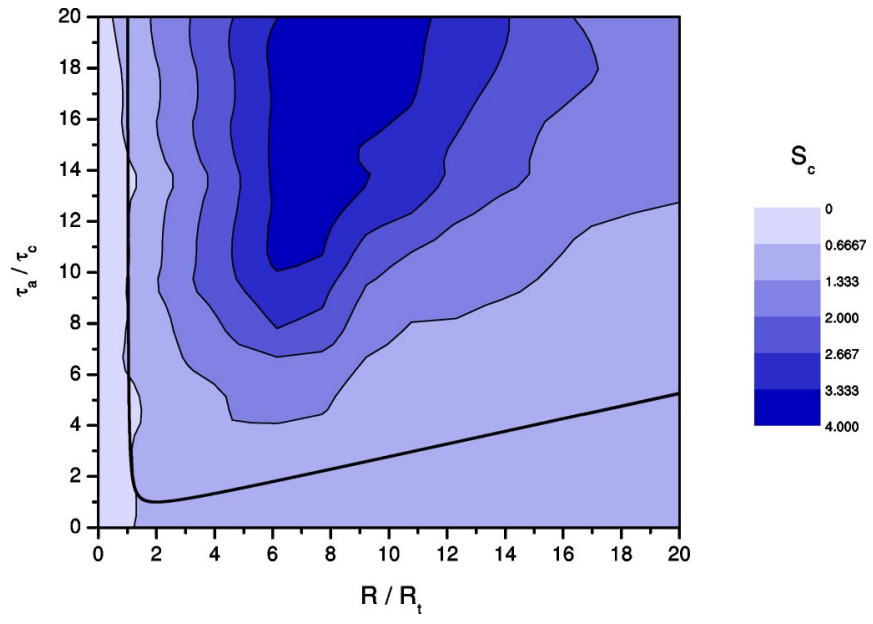

FIG. 4. Contour plot of the Shannon entropy of the distribution of the number of laser photons for a fixed value of $\tau_{c}=10$. Low values of $S_{c}$ (bright zones) indicate that the response of the system is nonoscillatory, while high values (dark zones) indicate an oscillatory response. The black line is the theoretical stability curve.

good indicator of the presence of oscillations in the system, as it must be low when the number of photons (or the population inversion) is essentially constant and high when this number oscillates. Figure 4 shows the presence of oscillations above and to the right of the theoretical stability curve (dark zones), where $\tau_{a}>\tau_{c}$ and $R>R_{t}$. On the other hand, in the bright zones, where $\tau_{a} \approx \tau_{c}$ or $R \leqslant R_{t}$, the number of laser photons does not oscillate. This is in good agreement with the theoretical predictions of Eq. (7), based on the linearization of the laser rate equations, and with the behavior observed in real systems. Laser spiking is characteristic of most solid-state and semiconductor lasers, which have a substantially longer decay time of the upper laser level than that of the photons in the laser cavity. This is not generally observed in gas lasers, whose decay times usually have the same order of magnitude.

Figures 5 and 6 show the temporal evolution of the num-

(a)

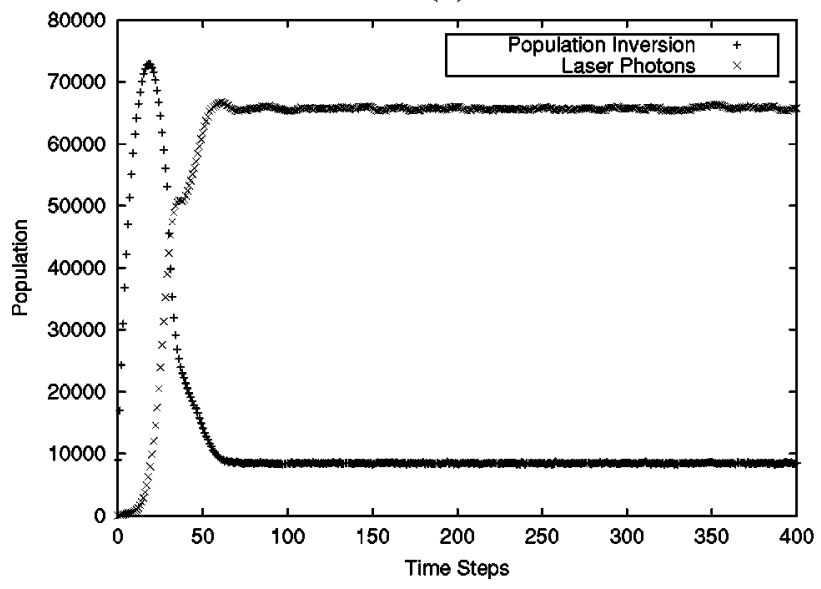

ber of laser photons and the population inversion for two different values of the system parameters, characteristic of the two different regimes in which it can operate. In this case, a lattice of $300 \times 300$ cells has been used in order to define the behavior more clearly. In both figures, the temporal evolution is shown at the left, and the evolution in a phase space with the number of laser photons versus the population inversion is shown at the right. Figure 5 corresponds to the parameter values for which the Shannon entropy is low (bright zones in Fig. 4) and, after an initial gain switching peak, the system shows a constant behavior. In phase space, after an initial transient the system goes to a fixed point. Figure 6 corresponds to the parameters values for which the Shannon entropy is high (dark zones in Fig. 4) and the system exhibits oscillations. In this particular case, the system shows damped oscillations which are associated to a spiral trajectory towards a steady state in phase space. For the values of the parameters corresponding to Fig. 6, a threshold pumping probability of $\lambda_{t}=0.0015$ is found. According to Eq. (A5), this means $\left(R / R_{t}\right)=\left(\lambda / \lambda_{t}\right)=(0.01 / 0.0015)$, which leads to a spiking period value predicted theoretically by Eq. (6) of $T_{s p}^{t h}=2 \pi / \omega_{s p}^{t h}=137.3$ time steps. The average spiking period of the oscillations resulting from our simulations is $T_{s p}^{s i m}=(127 \pm 10)$ time steps, which is in good agreement with the theoretical value predicted by Eq. (6).

The CA model is also very useful for observing the evolution of spatiotemporal patterns. Recently, quasiinstantaneous transverse patterns in a broad aperture laser have been measured for the first time [13]. In the case of a $\mathrm{CO}_{2}$ laser, a disordered distribution of spots, nonreproducible from shot to shot, which yields a boundary-determined regular structure when integrated in progressively longer time windows, has been found.

Figure 7 shows some snapshots of the instantaneous transverse patterns shown by the laser photon population in the lattice. Black color represents a cell with no photon present, gray represents cells with one photon, and white represents cells with two or more photons. Figures 7(a) and 7(b) corre-

(b)

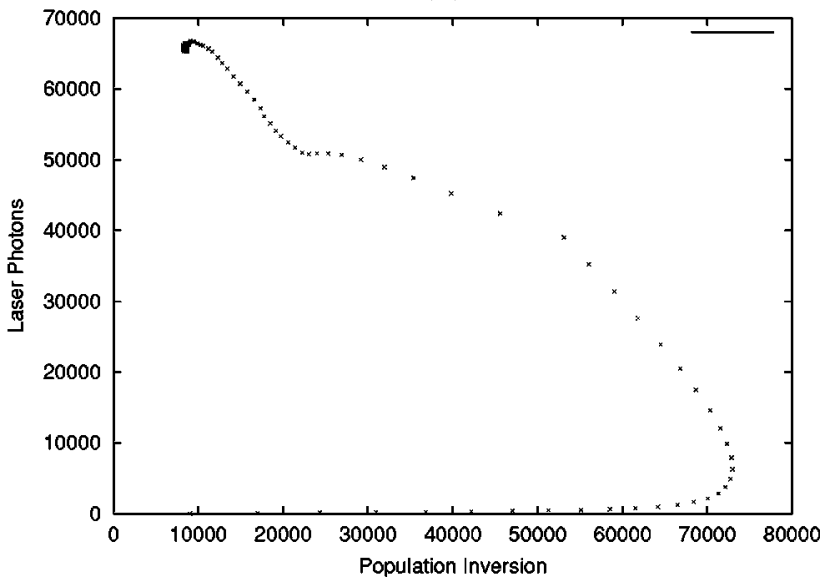

FIG. 5. Evolution of the system for the parameters: $\lambda=0.1, \tau_{c}=8, \tau_{a}=30$, where $\tau_{c}$ and $\tau_{a}$ are measured in time steps. Lattice: 300 $\times 300$ cells. (a) Number of laser photons and population inversion versus time. (b) Evolution in a phase space formed by the number of laser photons versus the population inversion. 
(a)

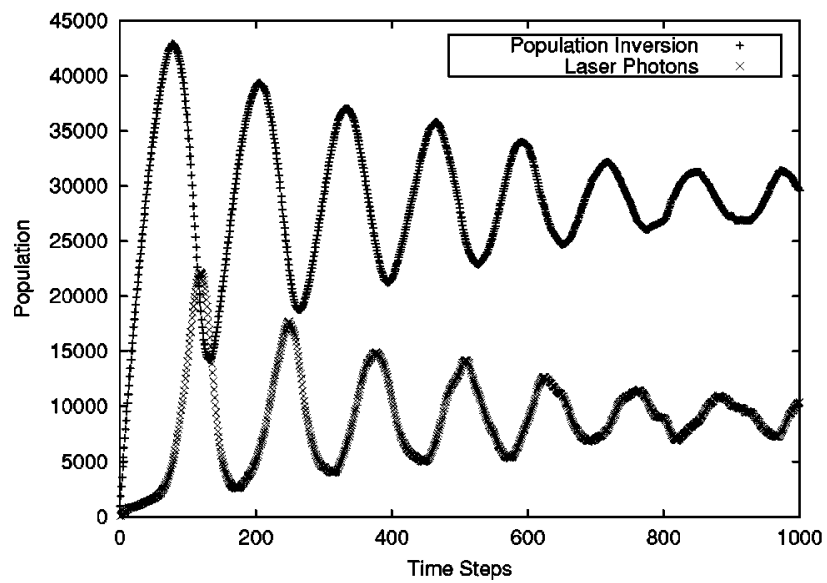

(b)

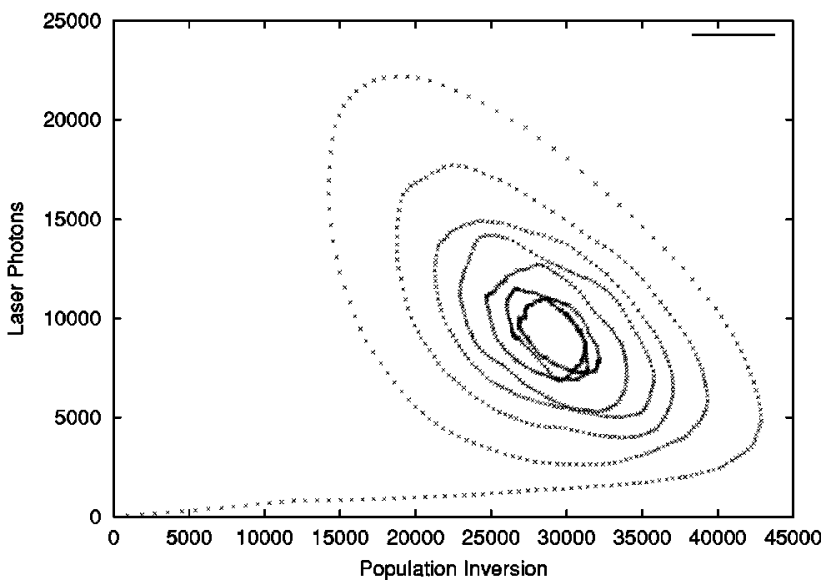

FIG. 6. Evolution of the system for the parameters: $\lambda=0.01, \tau_{c}=14, \tau_{a}=160$, where $\tau_{c}$ and $\tau_{a}$ are measured in time steps. Lattice: $300 \times 300$ cells. (a) Number of laser photons and population inversion versus time. (b) Evolution in phase space.

spond to the oscillatory behavior after a transient time. In this case, the system shows spatial structures that evolve in time resembling wave fronts that interact destructively with each other. The typical scale of these patterns is dependent on the value of the parameters, as can be seen by comparing Figs. 7(a) and 7(b). Figures 7(c) and 7(d) correspond to the nonoscillatory behavior and both figures have the same parameters values. Figure $7(\mathrm{c})$ was taken at time $t=50$ time (a)

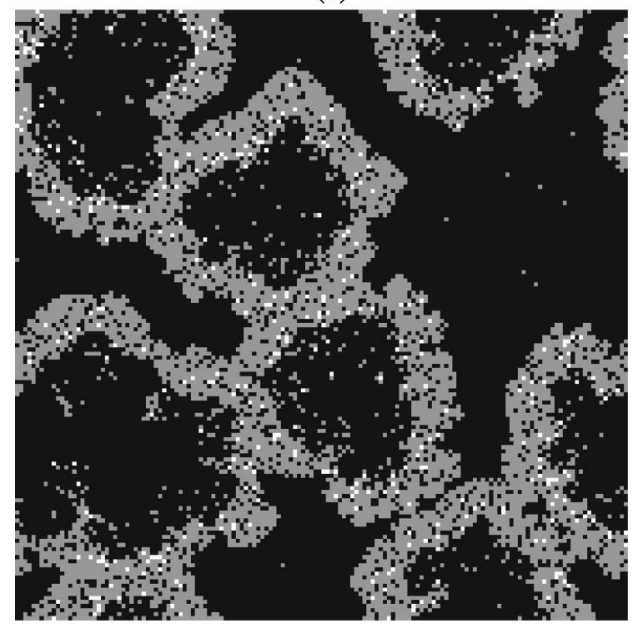

(c)

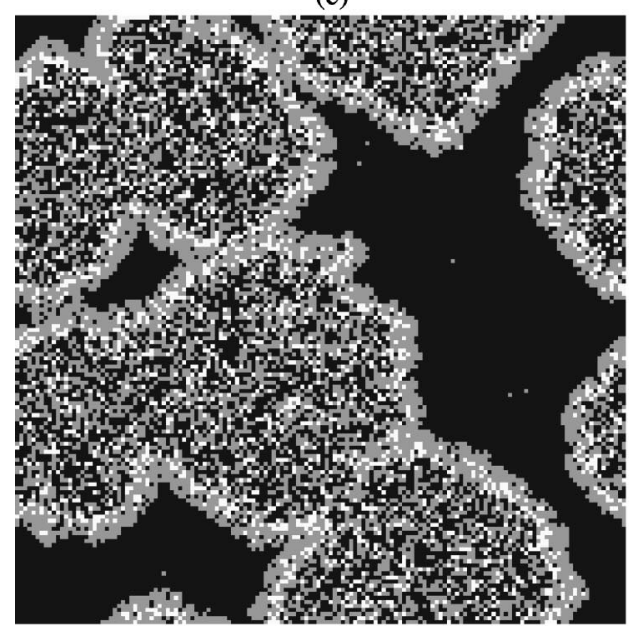

(b)

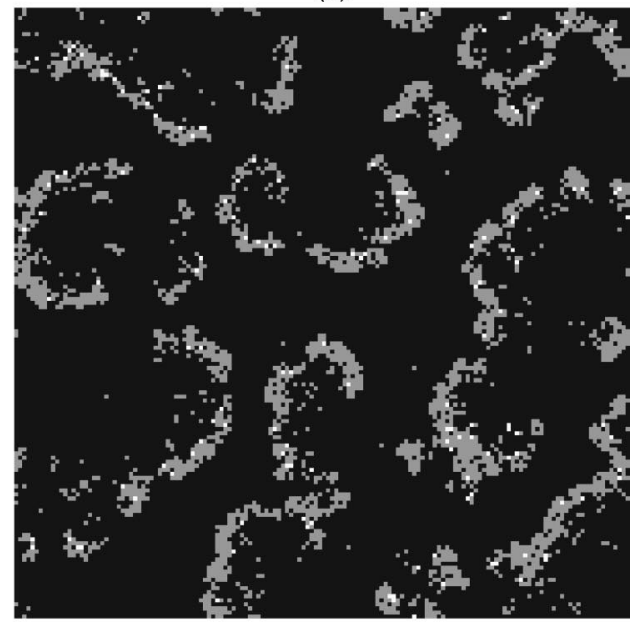

(d)

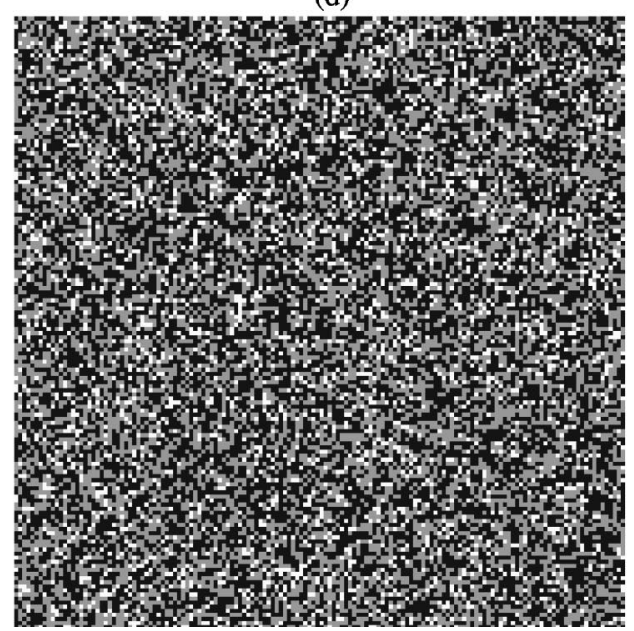

FIG. 7. Instantaneous transverse spatiotemporal patterns shown by the laser photon population in the system. (a) and (b) correspond to oscillatory behavior, whereas (c) and (d) correspond to nonoscillatory behavior. The values of the parameters are (a) $\lambda$ $=0.01, \tau_{c}=14, \tau_{a}=160$; (b) $\lambda$ $=0.03, \tau_{c}=6, \tau_{a}=150 ;$ (c) $\lambda$ $=0.1, \tau_{c}=8, \tau_{a}=30, t=50$; (d) $\lambda=0.1, \tau_{c}=8, \tau_{a}=30, t=200$. $\tau_{c}, \tau_{a}$, and $t$ are measured in time steps. 
steps, whereas Fig. 7(d) at time $t=200$ time steps. In this case, it can be observed that the laser process is activated initially with expanding avalanches and then reaches a stationary state at which the laser photons distribution is homogeneous.

\section{DISCUSSION}

Depending on the values of the three parameters $\tau_{a}, \tau_{c}, \lambda$ of the system, our simulations show that two distinct characteristic behaviors appear. The Shannon entropy of the laser photons distribution and of the population inversion allows us to obtain the parameter ranges in which each behavior takes place.

A high Shannon entropy (dark zones in Fig. 4) indicates a greater dispersion in the populations than in other areas of the parameters space. For these values of the parameters, the CA model reproduces the typical kind of behavior known as laser spiking - the temporal evolution of the populations of laser photons and inversion electrons oscillate in a correlated way, as shown in Fig. 6(a). A series of sharp, narrow "spikes" can be observed, whose amplitude decreases with time. In the phase space description [Fig. 6(b)], the system follows a spiral trajectory which converges towards a steadystate limit point.

On the other hand, a low Shannon entropy (bright zones in Fig. 4) indicates a smaller dispersion in the populations. For these parameter values, the behavior shown by the CA model corresponds to a constant regime - after an initial transient, the total number of laser photons and inversion electrons remain approximately constant, as is shown in Fig. 5(a). In the phase space description, Fig. 5(b), after a short transient the system reaches a fixed point.

Our model is successful in showing either one or the other of these two regimes, depending on the characteristic decay times involved. Laser spiking appears generally in situations in which the lifetime of the electrons in the excited state is substantially longer than the lifetime of the laser photons, whereas the constant regime mostly appears when both lifetimes are of the same order of magnitude.

The CA model can be applied during the interesting period of strong spiking, for which there is no simple analytic solution for the laser rate equations and the traditional approach relies on the numerical integration of the system of differential equations.

In Fig. 7, we can observe that the kind of spatiotemporal transverse patterns shown by the laser photons population depend on the population dynamics regime. For the laser spiking regime, spatiotemporal structures appear in the form of propagating wave fronts, Figs. 7(a) and 7(b). The characteristic scale of these structures depends on the three parameters of the system. It is remarkable that our rather simple and generic laser model produces spatiotemporal structures similar to those observed experimentally and with the numerical integration of very detailed Maxwell-Bloch equations models $[13,14]$.

On the other hand, when the system is in the constant regime, there is a homogeneous distribution of laser photons, Fig. 7(d). This is in agreement with the approximately con- stant values shown in the total number of laser photons. The laser process is initially activated with expanding avalanches, as shown in Fig. 7(c). When the avalanches have spread throughout the whole system, the constant steady state is reached.

In conclusion, we have presented here a CA model for the simulation of the population dynamics in a laser cavity. While simplified, the model is able to capture the essential features and phenomenology encountered in lasers such as relaxation oscillations, spiking behavior, and pattern formation. The CA approach for lasers is a very promising tool that can supplement the traditional approach based on the solution of sets of coupled differential equations. Its performance is very good, so that the simulation can be carried out on large lattices. The full parallelism of the CA can be implemented on special purpose computers or in computers with full parallel architectures, and therefore an important saving in computer time can be obtained. A CA model can represent an advantage in cases in which the system of differential equations have convergence problems, for example, stiff differential equations. The Maxwell-Bloch equations that describe many laser systems are of this type. Our model is free from this kind of convergence problems, so it can be very useful for this kind of lasers. However, work must be done in order to test this potential application, including a quantitative comparison of the computing times of the CA model versus the numerical integration of the full set of MaxwellBloch equations.

In the current state of our work, we see no obstacle to fine tune the proposed CA model in order to meet the characteristics of any particular laser system. This must be addressed in the near future. Additionally, we hope that similar models can be used to study other problems of current interest, such as chaotic laser dynamics [15] or the dynamics of a twophoton laser [16].

\section{ACKNOWLEDGMENT}

J. L. Guisado would like to thank Ramon Risco for helpful suggestions and discussions.

\section{APPENDIX: RELATIONSHIP BETWEEN THE PUMPING RATE $R$ AND THE PUMPING PROBABILITY $\lambda$}

Let us see how the pumping rate $R$, included in the laser rate equations, is related to the pumping probability $\lambda$, introduced in the CA model. $R$ is defined as the number of electrons that are excited from the ground state $E_{0}$ to the state $E_{3}$ per unit time, considered as a constant in the rate equations:

$$
R=\frac{d n_{3}}{d t} \Rightarrow n_{3}=R t .
$$

In the CA model, pumping is introduced as a probabilistic process, where $\lambda$ is the pumping probability per unit time of an electron from state $E_{0}$ to $E_{3}$. This means that if there are $n_{0}$ electrons in $E_{0}$, then the number of excitations in a time interval $d t$ will be $n \lambda d t$. So the population of state $E_{0}$ decreases as 


$$
d n_{0}=-\lambda d t n_{0} \quad \Rightarrow \quad n_{0}=n_{0_{i}} e^{-\lambda t}
$$

If we assume that $n_{3}$ is initially unpopulated, then $n_{0_{i}}$ $=n_{0}+n_{3}$ and the population of $E_{3}$ will increase with pumping as

$$
n_{3}=n_{0_{i}}-n_{0}=n_{0_{i}}\left(1-e^{-\lambda t}\right)
$$

Then, the pumping rate $R$ will be

$$
R=\frac{d n_{3}}{d t}=\lambda n_{0_{i}} e^{-\lambda t} .
$$

In general, if the only process in the system is the pumping, $R$ will decrease exponentially with time as the population of electrons in $E_{0}$ decreases. But the electrons pumped to level $E_{3}$ decay down again to level $E_{0}$ repopulating the ground level, and (as long as the pumping probability is small) after a transient period, $R$ stabilizes to a constant value:

$$
R=\lambda n_{0_{c}} \simeq \text { const, }
$$

where $n_{0_{c}}$ stands for the approximately constant value toward which the population of level $E_{0}$ tends. So after a transient period, there is a linear relationship between the pumping probability considered in our model and the constant pumping rate considered in the laser rate equations. This means that the pumping probabilities from the CA model must follow Eq. (5) with the exception of the value of the constant $K$ :

$$
\lambda_{t}=\frac{1}{K^{\prime} \tau_{a} \tau_{c}} .
$$

[1] H. Haken, Synergetics: An Introduction. Nonequilibrium Phase Transitions and Self-organization in Physics, Chemistry and Biology (Springer-Verlag, Berlin, 1983).

[2] A. Siegman, Lasers (University Science Books, Mill Valley, CA 1986).

[3] O. Svelto, Principles of Lasers (Plenum Press, New York, 1989).

[4] S. Wolfram, Physica D 10, 1 (1984).

[5] S. Wolfram, Cellular Automata and Complexity (AddisonWesley, New York, 1994).

[6] B. Chopard and M. Droz, Cellular Automata Modeling of Physical Systems (Cambridge University Press, Cambridge, 1998).

[7] T. Toffoli and N. Margolus, Cellular Automata Machines: A New Environment for Modelling (MIT, Cambridge, MA, 1987).
[8] G. Vichniac, Physica D 10, 96 (1984).

[9] B. Chopard, P. Luthi, and M. Droz, Phys. Rev. Lett. 72, 1384 (1994).

[10] A.K. Gunstensen, D.H. Rothman, S. Zaleski, and G. Zanetti, Phys. Rev. A 43, 4320 (1991).

[11] J. Gravner and D. Griffeath, Adv. Appl. Math. 21, 241 (1998).

[12] P. Seybold, L. Kier, and C.-K. Cheng, Int. J. Quantum Chem. 75, 751 (1999).

[13] F. Encinas-Sanz, I. Leyva, and J.M. Guerra, Phys. Rev. A 62, 043821 (2000).

[14] F. Encinas-Sanz, I. Leyva, and J.M. Guerra, Phys. Rev. Lett. 84, 883 (2000).

[15] G. Huyet and J. Tredicce, Physica D 96, 209 (1996).

[16] O. Pfister, W.J. Brown, M.D. Stenner, and D.J. Gauthier, Phys. Rev. Lett. 86, 4512 (2001). 